\title{
Moment Bounds for the Solutions of the Smoluchowski Equation with Coagulation and Fragmentation
}

\author{
Fraydoun Rezakhanlou* \\ UC Berkeley \\ Department of Mathematics \\ Berkeley, CA 94720-3840
}

December 4, 2009

\begin{abstract}
We prove various $L^{p}\left(\mathbb{R}^{d} \times[0, T]\right)$ bounds on moments $X_{a}(x, t):=\sum_{m \in \mathbb{N}} m^{a} f_{m}(x, t)$, (respectively $\int_{0}^{\infty} m^{a} f_{m}(x, t) d m$,) where $f_{m}$ is a solution of the discrete (respectively continuous) Smoluchowski coagulation-fragmentation equations with diffusion. In a previous paper [HR1] we proved similar results for all weak solution to the discrete Smoluchowski's equation provided that there is no fragmentation and certain moments are bounded in suitable $L^{q}$-spaces initially. In this paper we prove the corresponding results in the case of the continuous Smoluchowski's equation. When there is also fragmentation, we need to assume that the solution $f$ is regular in the sense that $f$ can be approximated by solutions to Smoluchowski equation for which the coagulation and fragmentation coefficients are 0 when the cluster sizes are large. We also need suitable assumptions on the coagulation rates to avoid gelation. On the fragmentation rate $\beta$, we assume that $\sup _{n} \sup _{m \leq \ell} \beta(m, n) / n<\infty$ for every positive $\ell$, and that there exist constants $a_{0} \geq 0$ and $c_{0}$ such that $\beta(n, m) \leq c_{0}(n+m)^{a_{0}}$.
\end{abstract}

\section{Introduction}

The Smoluchowski equation is a coupled system of partial differential equations that describes the evolving densities of a system of diffusing particles that are prone to coagulate

*This work is supported in part by NSF Grant DMS-0707890. 
in pairs and fragment into pairs. A sequence of functions $f_{n}: \mathbb{R}^{d} \times[0, \infty) \rightarrow[0, \infty), n \in \mathbb{N}$, is a solution of the discrete Smoluchowski equation if it satisfies

$$
\frac{\partial}{\partial t} f_{n}(x, t)=d(n) \Delta f_{n}(x, t)+Q_{n}(f)(x, t)
$$

with $Q_{n}=Q_{n}^{C}+Q_{n}^{F}, Q_{n}^{C}=Q_{n}^{C,+}-Q_{n}^{C,-}, Q_{n}^{F}=Q_{n}^{F,+}-Q_{n}^{F,-}$, where

$$
\begin{aligned}
& Q_{n}^{C,+}(f)(x, t)=\sum_{m=1}^{n-1} \alpha(m, n-m) f_{m}(x, t) f_{n-m}(x, t), \\
& Q_{n}^{C,-}(f)(x, t)=2 \sum_{m=1}^{\infty} \alpha(n, m) f_{n}(x, t) f_{m}(x, t), \\
& Q_{n}^{F,+}(f)(x, t)=2 \sum_{m=1}^{\infty} \beta(n, m) f_{n+m}(x, t),
\end{aligned}
$$

and

$$
Q_{n}^{F,-}(f)(x, t)=\sum_{m=1}^{n-1} \beta(m, n-m) f_{n}(x, t) .
$$

We will interpret this solution in a weak sense. Namely, we assume that $Q_{n}^{C, \pm}, Q_{n}^{F, \pm} \in$ $L^{1}\left(\mathbb{R}^{d} \times(0, T]\right)$ for each $T \in[0, \infty)$ and $n \in \mathbb{N}$, and that

$$
f_{n}(x, t)=S_{t}^{d(n)} f_{n}^{0}(x)+\int_{0}^{t} S_{t-s}^{d(n)} Q_{n}(x, s) d s
$$

where $\left\{f_{n}^{0}: n \in \mathbb{N}\right\}$ denotes the initial data, $S_{t}^{D}$ the semigroup associated with the equation $u_{t}=D \Delta u$, and where $Q_{n}(x, s)$ means $Q_{n}(f)(x, s)$.

In the continuous case, the summations $\sum_{m=1}^{n-1}$, and $\sum_{m=1}^{\infty}$, are replaced with $\int_{0}^{n} d m$, and $\int_{0}^{\infty} d m$, respectively. Similarly, a function $f: \mathbb{R}^{d} \times(0, \infty) \times[0, \infty) \rightarrow[0, \infty), f_{n}(x, t)=$ $f(x, n, t)$, is a solution to the continuous Smoluchowski equation if $f_{n}, Q_{n}^{C, \pm}, Q_{n}^{F, \pm} \in L^{1}\left(\mathbb{R}^{d} \times\right.$ $[0, T])$, for each $T, n \in(0, \infty)$, and $(1.2)$ holds.

In Hammond-Rezakhanlou [HR3], [HR2] and, Yaghouti-Rezakhanlou-Hammond[YRH] the equation (1.1) was derived from a microscopic model of coagulating Brownian particles which of course corresponds to the case $\beta \equiv 0$. In these articles we needed to make suitable assumptions on the microscopic details of the model that led to the property

$$
\lim _{n+m \rightarrow \infty} \frac{\alpha(n, m)}{(n+m)(d(n)+d(m))}=0
$$


of the macroscopic coagulation rate $\alpha$. This is our principal assumption on $\alpha$ in this article. The existence of solutions to (1.1) under the assumption

$$
\lim _{n \rightarrow \infty} \frac{\alpha(n, m)}{n}=0
$$

has been established in Wrzosek [W1], [W3], [MR] and Laurençot-Mischler [LM2], and in [LM1] when the equation (1.1) is formulated in a bounded domain. In this case one can prove the existence of a solution by first replacing $\alpha$ and $\beta$ by a suitable cutoff rates $\alpha^{(N)}$ and $\beta^{(N)}$, and pass to the limit. More precisely, $\alpha^{(N)}$ and $\beta^{(N)}$ are defined by

$$
\begin{gathered}
\alpha^{(N)}(n, m)= \begin{cases}\alpha(n, m) & \text { if } n \text { and } m \leq N, \\
0 & \text { otherwise }\end{cases} \\
\beta^{(N)}(n, m)= \begin{cases}\beta(n, m) & \text { if } n+m \leq N, \\
0 & \text { otherwise. }\end{cases}
\end{gathered}
$$

We can readily see that for such coagulation and fragmentation rates, there exists a unique solution $f^{(N)}$ for a given initial data. We then show that such a sequence $\left\{f^{(N)}\right\}_{N \in \mathbb{N}}$ has a convergent subsequence in $L^{1}$-sense and that each limit point $f$ is a weak solution to (1.1). We say a solution $f$ to (1.1) is regular if $f$ is obtained by the above approximation procedure.

Our results are valid for each dimension $d \geq 1$. Our main results would depend on some regularities in the initial data, and some assumptions on the parameters of the system. We now state the various assumptions that we require. As for the coagulation, fragmentation and diffusion parameters we consider four sets of assumptions.

Hypothesis 1.1 The function $d(\cdot)$ is positive and uniformly bounded. Moreover,

$$
\lim _{n+m \rightarrow \infty} \frac{\alpha(n, m)}{(n+m)(d(n)+d(m))}=0 .
$$

More precisely, for every $\delta>0$, there exists $k_{0}=k_{0}(\delta)>0$ such that if $n+m>k_{0}$, then

$$
\alpha(n, m) \leq \delta(n+m)(d(n)+d(m)) .
$$

Hypothesis 1.2 The function $d(n)$ is a non-increasing function of $n$ and that $\alpha(n, m) \leq$ $C_{0}(n+m)$ for a constant $C_{0}$. Moreover, there exist positive constants $r_{1}$ and $r_{2}$ and nonnegative constants $b_{2}$ and $b_{1}$ with $b_{2} \leq b_{1}, b_{1}>d / 2$, such that,

$$
r_{1} n^{-b_{1}} \leq d(n) \leq r_{2} n^{-b_{2}}
$$

for every $n>0$. In the case of the continuous Smoluchowski's equation, we also assume that $d(0)=\sup _{n} d(n)<\infty$. 
Hypothesis 1.3 For every $\ell>0$, there exists a constant $c_{1}(\ell)$ such that for every $m$ and $n$ with $m \leq \ell$,

$$
\beta(n, m) \leq c_{1}(\ell) n .
$$

Hypothesis 1.4 There exist constants $a_{0} \in[0, \infty)$ and $c_{0}$ such that

$$
\beta(n, m) \leq c_{0}(n+m)^{a_{0}} .
$$

Our notation for the various moments of $f$ in the discrete case will be

$$
X_{a}=X_{a}(x, t)=\sum_{n} n^{a} f_{n}(x, t), \quad \hat{X}_{a}=\hat{X}_{a}(x, t)=\sum_{n} n^{a} d(n)^{d / 2} f_{n}(x, t) .
$$

and

$$
\begin{aligned}
& Y_{a}(x, t)=\sum_{n, m} n m\left(n^{a}+m^{a}\right)(d(n)+d(m)) f_{n}(x, t) f_{m}(x, t), \\
& \hat{Y}_{a}(x, t)=\sum_{n, m}\left(n^{a} m+m^{a} n\right) \alpha(n, m) f_{n} f_{m} .
\end{aligned}
$$

In the continuous case all the summations are replaced with integration over $n, m \in(0, \infty)$. We also set

$$
\phi_{0}(x)= \begin{cases}|x|^{2-d} & \text { if } d \geq 3 \\ -\frac{1}{2 \pi} \log |x| \mathbb{1}(|x| \leq 1) & \text { if } d=2 \\ \frac{1}{2}(1-|x|) \mathbb{1}(2|x| \leq 1) & \text { if } d=1\end{cases}
$$

We now state the three theorems.

Theorem 1.1 Assume Hypotheses 1.1 and 1.3. Then for every $a \geq 2$ and positive $A$ and $T$, there exists a constant $C=C(a, A, T)$ such that, if $f$ is a regular solution and

$$
\iint X_{a}(x, 0) X_{1}(y, 0) \phi_{0}(x-y) d x d y \leq A,
$$

and

$$
\mathrm{e} s \sup _{x} \int\left(X_{a}+X_{0}\right)(y, 0) \phi_{0}(x-y) d y \leq A, \quad \int\left(X_{a}+X_{0}\right)(x, 0) d x \leq A
$$

then

$$
\int_{0}^{T} \int Y_{a-1} d x d t \leq C, \quad \int_{0}^{T} \int \hat{Y}_{a-1} d x d t \leq C
$$

and

$$
\sup _{t \in[0, T]} \int X_{a}(x, t) d x \leq C .
$$

Moreover, when there is no fragmentation, our results are valid for every weak solution. 
Theorem 1.2 Assume Hypotheses 1.2 and 1.4. Given $\left(b_{1}, b_{2}, a_{0}\right)$ as in those Hypotheses, there exists a function $\gamma\left(\cdot ; b_{1}, b_{2}, a_{0}\right):(1, \infty) \rightarrow(0, \infty)$ with $\lim _{a \rightarrow \infty} \gamma\left(a ; b_{1}, b_{2}, a_{0}\right)=\infty$ such that if $f$ is a regular solution to discrete (respectively continuous) Smoluchowski equation and

$$
\begin{aligned}
& \sum_{n} n^{\gamma}\left\|f_{n}^{0}\right\|_{L^{\infty}\left(\mathbb{R}^{d}\right)}<\infty, \quad(\text { respectively } \\
& X_{a} \in L^{1}\left(\mathbb{R}^{d} \times[0, T]\right), \quad\left(\text { respectively } X_{a}, X_{a_{0}} \in L^{1}\left(\mathbb{R}^{d} \times[0, T]\right),\right)
\end{aligned}
$$

then

$$
\sum_{n} n^{\gamma}\left\|f_{n}\right\|_{L^{\infty}\left(\mathbb{R}^{d} \times[0, T]\right)}<\infty, \quad\left(\text { respectively } \int_{0}^{\infty}(n+1)^{\gamma}\left\|f_{n}^{0}\right\|_{L^{\infty}\left(\mathbb{R}^{d} \times[0, T]\right)}<\infty,\right)
$$

where $\gamma=\gamma\left(a ; b_{1}, b_{2}, a_{0}\right)$. Moreover, when there is no fragmentation, our results are valid for every weak solution.

Remark 1.1 In particular, if Hypotheses 1.1-1.4 hold, $X_{a}(\cdot, 0) \in L^{1}\left(\mathbb{R}^{d}\right)$, and

$$
\sum_{n} n^{a}\left\|f_{n}^{0}\right\|_{L^{\infty}\left(\mathbb{R}^{d}\right)}<\infty
$$

for every $a \in \mathbb{N}$, then $X_{a} \in L^{\infty}\left(\mathbb{R}^{d} \times[0, T]\right) \cap L^{1}\left(\mathbb{R}^{d} \times[0, T]\right)$ for every $a \in \mathbb{N}$ and $T \in(0, \infty)$. We refer to (3.16) for the explicit form of the function $\gamma$. Also note that Theorem 1.1 offers sufficient conditions to ensure $X_{a} \in L^{1}\left(\mathbb{R}^{d} \times[0, T]\right.$ ) (so that this theorem has been invoked in Remark 1.1).

We end this section with two results which were carried out in [HR1] in the discrete case. The case of continuous Smoluchowski equation can be treated in a similar way. Note that the uniqueness result is a straightforward generalization of the uniqueness theorem of Ball and Carr $[\mathrm{BC}]$.

Theorem 1.3 (Conservation of Mass) Let $f$ be a regular solution of (1.1). Assume that (1.13) holds for $a=2$. Then $f$ conserves mass on the time interval $[0, T]$. Moreover, when there is no fragmentation, our results are valid for every weak solution.

Theorem 1.4 (Uniqueness) Suppose that there is no fragmentation and that, for some $c_{0}>0$, we have that $\alpha(n, m) \leq c_{0} n m$ for each $n, m \in \mathbb{N}$. Then there is a unique weak solution of (1.1) on the interval $[0, T]$ among those satisfying $X_{2} \in L^{\infty}\left(\mathbb{R}^{d} \times[0, T]\right)$.

For some related works, we refer to $[\mathrm{A}],[\mathrm{AW}]$ (local exitence and uniqueness), [W2] (uniqueness and mass conservation for bounded $\alpha$ and almost constant diffusion coefficient), and $[\mathrm{N}]$ (uniqueness and existence for an example with unbounded diffusion coefficient). 
On account of Theorem 1.2, we have that $X_{2} \in L^{\infty}$ if the same is true initially and for some (possibly large) $a$, we have that $X_{a} \in L^{1}$. On the other hand Theorem 1.1 guarrantees that $X_{a} \in L^{1}$ under suitable assumptions on the initial conditions. In summary, there would be a unique solution to (1.1) if there is no fragmentation, $X_{2} \in L^{\infty}$ initially, and certain moments of initial data is bounded with respect to the norms given in Theorem 1.1.

\section{Proof of Theorem 1.1}

We only give a proof of Theorem 1.1 in the case of the discrete Smoluchowski equation because the continuous case can be treated by verbatim arguments.

The case $\beta \equiv 0$ has already been established for all weak solutions in [HR1]. It remains to establish Theorem 1.1 for regular solutions. Since we are considering regular solutions only, it suffices to establish Theorem 1.1 when $\alpha(n, m)=0$ for $n$ or $m>N$ and $\beta(n, m)=0$ for $(n, m)$ satisfying $n+m>N$. (Of course our constants will not depend on the cutoff parameter $N$.) Observe that under such assumptions on $\alpha$ and $\beta$, we always have

$$
\sum_{1}^{l} n Q_{n}=0
$$

for $l \geq 2 N$, because

$$
\sum_{n=1}^{l} n Q_{n}=\sum_{n, m}[n \mathbb{1}(n \leq l<n+m)+m \mathbb{1}(m \leq l<n+m)]\left(\beta(n, m) f_{n+m}-\alpha(n, m) f_{n} f_{m}\right) .
$$

As an immediate consequence of (2.1), we have

$$
\int \sum_{n=1}^{l} n f_{n}(x, t) d x=\int \sum_{n=1}^{l} n f_{n}(x, 0) d x .
$$

We first recall a lemma from [HR1].

Lemma 2.1 There exist functions $H$ and $K$ such that $H \geq 0, K$ is bounded, $K$ is of compact support,

$$
-\Delta H(x)=\delta_{0}-K(x),
$$

weakly, and the function $H-\phi_{0}$ is bounded. (The function $\phi_{0}$ was defined in (1.10).)

In fact when $d \geq 3$, we can choose $K \equiv 0$ and $H(x)=c(d)|x|^{2-d}$ with $c(d)=(d-2)^{-1} w_{d}^{-1}$ where $w_{d}$ denotees the $(d-1)$-dimensional measure of the unit sphere. We can not afford $K \equiv 0$ in (2.1) when $d=1$ or 2 , and more work is needed for the construction of $H$. We refer the reader to [HR1] for the proof of Lemma 2.1. As our next preparation for the proof of Theorem 1.1, we state and prove Lemma 2.2 which is the analog of Lemma 3.2 of [HR1]. 
Lemma 2.2 Let $H$ be as in Lemma 2.1. Then there exists a constant $c_{1}$ such that for every regular solution $f$,

$$
\sup _{x} \sum_{n=1}^{l} n\left(f_{n}^{\delta} *_{x} H\right)(x, t) \leq \sup _{x} \sum_{n=1}^{l} n\left(f_{n}^{\delta} *_{x} H\right)(x, 0)+c_{1} t \int \sum_{n=1}^{l} n f_{n}(x, 0) d x
$$

for every $\delta>0, l \geq 2 N$ and $t>0$. Here $f^{\delta}=f *_{x} \zeta^{\delta}$ where $\zeta^{\delta}(z)=\delta^{-d} \zeta\left(\frac{z}{\delta}\right)$ with $\zeta$ a nonnegative smooth function of compact support and total integral 1 . The constant $c_{1}$ can be chosen to be 0 when $d>2$.

Proof. We certainly have

$$
\begin{aligned}
\sum_{1}^{l} n\left(f_{n}^{\delta} *_{x} H\right)(x, t)= & \sum_{1}^{l} n\left(f_{n}^{\delta} *_{x} H\right)(x, 0)-\int_{0}^{t} \sum_{1}^{l} n d(n) f_{n}^{\delta}(x, s) d s \\
& +\int_{0}^{t} \sum_{n=1}^{l} n d(n)\left(f_{n}^{\delta} *_{x} K\right)(x, s) d s+\int_{0}^{t} \sum_{n=1}^{l} n\left(Q_{n, \delta} *_{x} H\right)(x, s) d s,
\end{aligned}
$$

where $Q_{n, \delta}=Q_{n} *_{x} \zeta^{\delta}$. Since $K$ and $d$ are bounded,

$$
\begin{aligned}
\left\|\sum_{n=1}^{l} n d(n)\left(f_{n}^{\delta} *_{x} K\right)(\cdot, s)\right\|_{L^{\infty}(d x)} & \leq c_{1}\left\|\sum_{n=1}^{l} n f_{n}^{\delta}(\cdot, s)\right\|_{L^{1}(d x)} \leq c_{1}\left\|\sum_{n=1}^{l} n f_{n}(\cdot, s)\right\|_{L^{1}(d x)}\left\|\zeta^{\delta}\right\|_{L^{1}(d x)} \\
& =c_{1}\left\|\sum_{n=1}^{l} n f_{n}(\cdot, 0)\right\|_{L^{1}(d x)}
\end{aligned}
$$

where we used (2.2) and $\left\|\zeta^{\delta}\right\|_{L^{1}(d x)}=1$ for the equality. The Lemma follows from this, (2.3) and (2.1).

Proof of Theorem 1.1. Assume that $l \geq 2 N$ and set

$$
\begin{aligned}
Z^{l, \delta}(t) & =\int\left(\sum_{n=1}^{l} n^{a} f_{n}^{\delta}(x, t)\right)\left(\sum_{n=1}^{l} n f_{n}^{\delta} *_{x} H(x, t)\right) d x \\
Z^{l}(t) & =\int\left(\sum_{n=1}^{l} n^{a} f_{n}(x, t)\right)\left(\sum_{n=1}^{l} n\left(f_{n} *_{x} H\right)(x, t)\right) d x .
\end{aligned}
$$

We have that weakly

$$
\frac{d}{d t} Z^{l, \delta}(t)=\Omega_{1}+\Omega_{2}+\Omega_{3}+\Omega_{4}+\Omega_{5}+\Omega_{6},
$$


where,

$$
\begin{aligned}
& \Omega_{1}=-\int\left(\sum_{n=1}^{l} n^{a} f_{n}^{\delta}(x, t)\right)\left(\sum_{n=1}^{l} n d(n) f_{n}^{\delta}(x, t)\right) d x \\
& \Omega_{2}=-\int\left(\sum_{n=1}^{l} n^{a} d(n) f_{n}^{\delta}(x, t)\right)\left(\sum_{n=1}^{l} n f_{n}^{\delta}(x, t)\right) d x \\
& \Omega_{3}=\int\left(\sum_{n=1}^{l} n^{a} f_{n}^{\delta}(x, t)\right)\left(\sum_{n=1}^{l} n\left(Q_{n, \delta} *_{x} H\right)(x, t)\right) d x \\
& \Omega_{4}=\int\left(\sum_{n=1}^{l} n^{a} Q_{n, \delta}(x, t)\right)\left(\sum_{n=1}^{l} n\left(f_{n}^{\delta} *_{x} H\right)(x, t)\right) d x \\
& \Omega_{5}=\int\left(\sum_{n=1}^{l} n^{a} f_{n}^{\delta}(x, t)\right)\left(\sum_{n=1}^{l} n d(n)\left(f_{n}^{\delta} *_{x} K\right)(x, t)\right) d x \\
& \Omega_{6}=\int\left(\sum_{n=1}^{l} n^{a} d(n) f_{n}^{\delta}(x, t)\right)\left(\sum_{n=1}^{l} n\left(f_{n}^{\delta} *_{x} K\right)(x, t)\right) d x,
\end{aligned}
$$

with $Q_{n, \delta}=Q_{n} *_{x} \zeta^{\delta}$. We can readily show

$$
\Omega_{1}+\Omega_{2}=-\frac{1}{2} \int \sum_{n, m} \mathbb{1}(n, m \leq l) n m(d(n)+d(m))\left(n^{a-1}+m^{a-1}\right) f_{n}^{\delta} f_{m}^{\delta} d x .
$$

By (2.1), we know that $\Omega_{3}=0$. By the boundedness of the functions $K(\cdot), d(\cdot)$, and $(2.2)$ we deduce

$$
\left|\Omega_{5}+\Omega_{6}\right| \leq c_{1} \int X_{a}^{l, \delta} d x
$$

where $X_{a}^{l, \delta}=X_{a}^{l} * \zeta^{\delta}$ and

$$
X_{a}^{l}=\sum_{n=1}^{l} n^{a} f_{n}
$$


It remains to bound $\Omega_{4}$. Note that

$$
\begin{aligned}
\sum_{n=1}^{l} n^{a} Q_{n} & =\sum_{n, m}\left[(n+m)^{a} \mathbb{1}(n+m \leq l)-n^{a} \mathbb{1}(n \leq l)-m^{a} \mathbb{1}(m \leq l)\right] \\
& \left(\alpha(n, m) f_{n} f_{m}-\beta(n, m) f_{n+m}\right) \\
\leq & \sum_{n, m}\left[(n+m)^{a} \mathbb{1}(n+m \leq l)-n^{a} \mathbb{1}(n \leq l)-m^{a} \mathbb{1}(m \leq l)\right] \alpha(n, m) f_{n} f_{m} \\
\leq & c_{2} \sum_{n, m}\left(n^{a-1} m+m^{a-1} n\right) \mathbb{1}(n+m \leq l) \alpha(n, m) f_{n} f_{m}=: c_{2} \hat{Y}_{a-1}^{l} .
\end{aligned}
$$

Hence, we may apply Lemma 2.2 and (??) to deduce

$$
\Omega_{4} \leq c_{2} \int \hat{Y}_{a-1}^{l, \delta}\left(\sum_{n=1}^{l} n\left(f_{n}^{\delta} *_{x} H\right)(x, t)\right) d x \leq c_{3} \int \hat{Y}_{a-1}^{l, \delta} d x
$$

where $\hat{Y}_{a-1}^{l, \delta}=\hat{Y}_{a-1}^{l} * \zeta^{\delta}$. We use Hypothesis 1.1 to assert that for every $\delta_{0}>0$, there exists $k_{0}=k_{0}\left(\delta_{0}\right)$ such that if $k>k_{0}$, then

$$
\begin{aligned}
\hat{Y}_{a-1}^{l} \leq & \left.\delta_{0} \sum_{n, m} \mathbb{1}(n+m \leq l)\right)\left(n^{a-1} m+m^{a-1} n\right)(n+m)(d(n)+d(m)) f_{n} f_{m} \\
& +2 k_{0}\left(\delta_{0}\right)^{a} \sum_{n, m} \mathbb{1}\left(n+m \leq k_{0}\left(\delta_{0}\right)\right) \alpha(n, m) f_{n} f_{m} .
\end{aligned}
$$

From $(2.4-8)$ we deduce,

$$
\begin{aligned}
\frac{d}{d t} Z^{l, \delta}(t) \leq & -\frac{1}{2} \int \sum_{n, m} \mathbb{1}(n, m \leq l) n m\left(n^{a-1}+m^{a-1}\right)(d(n)+d(m)) f_{n}^{\delta} f_{m}^{\delta} d x \\
& +2 c_{3} \delta_{0} \int \sum_{n, m} \mathbb{1}(n, m \leq l) n m\left(n^{a-1}+m^{a-1}\right)(d(n)+d(m))\left(f_{n} f_{m}\right) * \zeta^{\delta} d x \\
& +2 c_{3} k_{0}\left(\delta_{0}\right)^{a} \int \sum_{n, m} \mathbb{1}\left(n+m \leq k_{0}(\delta)\right) \alpha(n, m)\left(\left(f_{n} f_{m}\right) * \zeta^{\delta}\right) d x+c_{1} \int X_{a}^{l, \delta} d x,
\end{aligned}
$$

where we used the inequality

$$
(n+m)\left(n^{a-2}+m^{a-2}\right) \leq 2\left(n^{a-1}+m^{a-1}\right),
$$

which is valid provided that $a \geq 2$. On the other hand,

$$
\frac{d}{d t} \int \sum_{n=1}^{k} n^{a} f_{n}(x, t) d x=\int \sum_{n=1}^{k} n^{a} Q_{n}(x, t) d x .
$$


Hence, by (2.6),

$$
\begin{aligned}
\int \sum_{n=1}^{k} n^{a} f_{n}(x, t) d x & \leq \int \sum_{n=1}^{k} n^{a} f_{n}(x, 0) d x+c_{4} \int_{0}^{t} \int \hat{Y}_{a-1}^{k}(x, s) d x d s \\
& \leq \int \sum_{n=1}^{k} n^{a} f_{n}(x, 0) d x+c_{4} \int_{0}^{t} \int \hat{Y}_{a-1}^{k}(x, s) d x d s .
\end{aligned}
$$

Sending $k \rightarrow \infty$ yields

$$
\int X_{a}(x, t) d x \leq \int X_{a}(x, 0) d x+c_{4} \int_{0}^{t} \int \hat{Y}_{a-1}(x, s) d x d s .
$$

One more time we use (2.8) to assert

$$
\begin{aligned}
\int X_{a}(x, t) d x \leq & c_{5}+c_{4} \delta_{0} \int_{0}^{t} \int Y_{a-1} d x d s \\
& +2 c_{4} k_{0}\left(\delta_{0}\right)^{a} \int_{0}^{t} \int \sum_{n, m} \mathbb{1}\left(n+m \leq k_{0}(\delta)\right) \alpha(n, m) f_{n} f_{m} d x d s .
\end{aligned}
$$

In (2.9) we let $\delta \rightarrow 0$ and $l \rightarrow \infty$, and then apply (2.11) to deduce that the expression $Z^{\infty}(t)-Z^{\infty}(0)$ is bounded above by

$$
\begin{aligned}
& \left(2 c_{3} \delta_{0}+4 c_{1} c_{2} t \delta_{0}-\frac{1}{2}\right) \int_{0}^{t} \int \sum_{n, m} n m\left(n^{a-1}+m^{a-1}\right)(d(n)+d(m)) f_{n} f_{m} d x d s \\
& +2\left(c_{1} c_{2} t+c_{3}\right) k_{0}\left(\delta_{0}\right)^{a} t \int_{0}^{t} \int \sum_{n, m} \mathbb{1}\left(n+m \leq k_{0}(\delta)\right) \alpha(n, m) f_{n} f_{m} d x d s .
\end{aligned}
$$

Let us simply write $k_{0}$ for $k_{0}\left(\delta_{0}\right)$. Note that from

$\frac{d}{d t} \int \sum_{n=1}^{k_{0}} f_{n} d x=\int\left[\mathbb{1}\left(n+m \leq k_{0}\right)-\mathbb{1}\left(n \leq k_{0}\right)-\mathbb{1}\left(m \leq k_{0}\right)\right]\left(\alpha(n, m) f_{n} f_{m}-\beta(n, m) f_{n+m}\right) d x$,

we learn that the expression

$$
\int_{0}^{t} \int \sum_{n, m \leq k_{0}} \alpha(n, m) f_{n} f_{m} d x d s
$$


is bounded above by

$$
\begin{aligned}
& \int \sum_{n=1}^{k_{0}} f_{n}(x, 0) d x-\int_{0}^{t} \int \sum_{n, m}\left[\mathbb{1}\left(n+m \leq k_{0}\right)-\mathbb{1}\left(n \leq k_{0}\right)-\mathbb{1}\left(m \leq k_{0}\right)\right] \beta(n, m) f_{n+m} d x d s \\
& \leq \int \sum_{n=1}^{k_{0}} f_{n}(x, 0) d x+\int_{0}^{t} \int 2 \sum_{n, m} \mathbb{1}\left(n \text { or } m \leq k_{0}\right) \beta(n, m) f_{n+m} d x \\
& \leq c_{5}+4 \int_{0}^{t} \int \sum_{r=1}^{\infty}\left[\sum_{n+m=r} \mathbb{1}\left(n \leq k_{0}\right) \beta(n, m)\right] f_{r} d x d s \\
& \leq c_{5}+4 k_{0} c_{1}\left(k_{0}\right) \int_{0}^{t} \int \sum_{r=1}^{\infty} r f_{r} d x d s,
\end{aligned}
$$

where for the last inequality we have used Hypothesis 1.3. From this and (2.2) we deduce,

$$
\int_{0}^{t} \int \sum_{n, m \leq k_{0}} \alpha(n, m) f_{n} f_{m} d x d s \leq c_{4}+c_{6}\left(k_{0}\right) t
$$

From this and (2.12) we learn that the expression $Z^{\infty}(t)-Z^{\infty}(0)$ is bounded above by

$$
\begin{aligned}
& \left(2 c_{3} \delta_{0}+4 c_{1} c_{2} t \delta_{0}-\frac{1}{2}\right) \int_{0}^{t} \int \sum_{n, m} n m\left(n^{a-1}+m^{a-1}\right)(d(n)+d(m)) f_{n} f_{m} d x d s \\
& \quad+c_{7}\left(k_{0}\left(\delta_{0}\right)\right)\left(1+t^{2}\right)
\end{aligned}
$$

for a constant $c_{7}(\cdot)$. We now choose $\delta_{0}=\delta_{0}(t)$ so that $\frac{1}{2}>\left(2 c_{3}+4 c_{1} c_{2} t\right) \delta_{0}$. With this choice the bounds in (1.13) follow with the help of (1.11). To prove (1.14), we simply use (2.11), (2.13) and (1.13).

\section{$3 \quad L^{\infty}$ bounds}

In this section we establish Theorem 1.2. To ease the notation, we do not display the dependence on the $x$-variable. For example we simply write $f_{n}(t)$ for $f_{n}(\cdot, t)$ and $Q_{n}(t)$ for 
$Q_{n}(\cdot, t)$. We first claim

$$
\begin{aligned}
\sum_{1}^{l} n d(n)^{d / 2} f_{n}(t) \leq & d(1)^{d / 2} S_{t}^{d(1)}\left(\sum_{1}^{l} n f_{n}^{0}\right) \\
& +d(l)^{d / 2} \int_{0}^{t} S_{t-s}^{d(l)}\left(\sum_{1}^{l} n Q_{n}^{C}(s)\right) d s \\
& +\int_{0}^{t} \sum_{1}^{l} n d(n)^{d / 2}\left(S_{t-s}^{d(n)} Q_{n}^{+, F}(s)\right) d s .
\end{aligned}
$$

The proof of (3.1) is identical to the proof of (4.2) of [HR1] and we do not repeat it here. However, the proof given in [HR1] uses induction and is not applicable in the continuous case. Here is the corresponding statement and its proof in the continuous case.

Lemma 3.1 Suppose that $f$ is a solution to the continuous Smoluchowski equation. Then for every $l \geq 0$, we have

$$
\begin{aligned}
\int_{0}^{l}(n+1) d(n)^{d / 2} f_{n}(t) d n \leq & d(0)^{d / 2} S_{t}^{d(0)}\left(\int_{0}^{l}(n+1) f_{n}^{0} d n\right) \\
& +d(l)^{d / 2} \int_{0}^{t} S_{t-s}^{d(l)}\left(\int_{0}^{l}(n+1) Q_{n}^{C}(s) d n\right) d s \\
& +\int_{0}^{t}\left[\int_{0}^{l}(n+1) d(n)^{d / 2}\left(S_{t-s}^{d(n)} Q_{n}^{+, F}(s)\right) d n\right] d s,
\end{aligned}
$$

almost everywhere, where $d(0)=\sup _{n} d(n)$.

Proof. First we rewrite (3.2) as

$$
A(l) \leq B(l)+C(l, l)+D(l),
$$

where

$$
\begin{aligned}
A(l) & =\int_{0}^{l}(n+1) d(n)^{d / 2} f_{n}(t) d n, \\
B(l) & =d(0)^{d / 2} S_{t}^{d(0)}\left(\int_{0}^{l}(n+1) f_{n}^{0} d n\right), \\
C(k, l) & =d(k)^{d / 2} \int_{0}^{t} S_{t-s}^{d(k)}\left(\int_{0}^{l}(n+1) Q_{n}^{C}(s) d n\right) d s, \\
D(l) & =\int_{0}^{t}\left[\int_{0}^{l}(n+1) d(n)^{d / 2} S_{t-s}^{d(n)} Q_{n}^{+, F}(s) d n\right] d s .
\end{aligned}
$$


Note that both sides of (3.2) are functions of $(x, t)$ and (3.2) is equivalent to saying

$$
A(l, J) \leq B(l, J)+C(l, l, J)+D(l, J),
$$

for every smooth function $J \geq 0$ of compact support, where $A(l, J), B(l, J), C(k, l, J)$, and $D(l, J)$, are obtained by multiplying $A(l), B(l), C(k, l)$ and $D(l)$, by $J$ and integrating with respect to $d x d t$. Set

$$
\eta(k, l)=B(l, J)+C(k, l, J)+D(l, J)-A(l, J), \quad \gamma(l)=\eta(l, l) .
$$

The inequality (3.4) means that $\gamma(l) \geq 0$ and it suffices to show that $\gamma$ is non-decreasing because $\gamma(0)=0$. This is equivalent to saying that the weak derivative of $\gamma$ is non-negative which would follow if we can show that $\eta$ is non-decreasing in $k$-variable and that $\frac{\partial \eta}{\partial l}(l, l) \geq 0$. The former is an immediate consequence of

$$
\int_{0}^{l}(n+1) Q_{n}^{C} d n \leq 0,
$$

the assumption that $d(\cdot)$ is non-increasing, and the elementary fact that the function $a \mapsto$ $a^{d / 2} S_{t}^{a} g$ is decreasing for every non-positive function $g$. For the latter observe that $\frac{\partial \eta}{\partial l}(l, l)$ equals

$$
\begin{aligned}
& d(0)^{d / 2}(l+1) \iint\left(S_{t}^{d(0)} f_{l}^{0}\right) J d x d t+d(l)^{d / 2}(l+1) \iiint_{0}^{t}\left(S_{t-s}^{d(l)} Q_{l}^{C}(s)\right) d s J d x d t \\
& \quad+d(l)^{d / 2}(l+1) \iiint_{0}^{t}\left(S_{t-s}^{d(l)} Q_{l}^{+, F}(s)\right) d s J d x d t-d(l)^{d / 2}(l+1) \iint f_{l} J d x d t \\
& \geq d(l)^{d / 2}(l+1) \iint S_{t}^{d(l)} f_{l}^{0} J d x d t+d(l)^{d / 2}(l+1) \iiint_{0}^{t} S_{t-s}^{d(l)} Q_{l}^{C}(s) d s J d x d t \\
& \quad+d(l)^{d / 2}(l+1) \iiint_{0}^{t}\left[S_{t-s}^{d(l)} Q_{l}^{+, F}(s)\right] d s J d x d t-d(l)^{d / 2}(l+1) \iint f_{l} J d x d t \\
& \geq 0,
\end{aligned}
$$

where for the first inequality we used the monotonicity of $d(\cdot)$ and the fact that the function $a \mapsto a^{d / 2} S_{t}^{a}$ is increasing, and for the second inequality we used (1.2) and $Q^{+, F} \geq Q^{F}$. This completes the proof.

Remark 3.1. When there is no fragmentation, the bounds (3.1) and (3.2), and the inequality (3.5) imply

$$
\begin{gathered}
\operatorname{esssup}_{x}\left(\sum_{n=1}^{\infty} n d(n)^{d / 2} f_{n}(x, t)\right) \leq d(1)^{d / 2} \operatorname{esssup}_{x}\left(\sum_{n=1}^{\infty} n f_{n}(x, 0)\right), \\
\operatorname{esssup}_{x}\left(\int_{0}^{\infty}(n+1) d(n)^{d / 2} f_{n}(x, t) d n\right) \leq d(0)^{d / 2} \operatorname{esssup}_{x}\left(\int_{0}^{\infty}(n+1) f_{n}(x, 0) d n\right),
\end{gathered}
$$


for the solutions to the Smoluchowski equation in the discrete and continuous case respectively. This yields a $L^{\infty}$ bound at time $t$ provided that the associated bound is assumed initially. This and the $L^{1}$ bound of Section 2 can be used to establish Theorem 1.2 when there is no fragmentation. This was carried out in Section 4 of [HR1] in the discrete case. A similar proof is applicable in the continuous case.

As our next step towards the proof of Theorem 1.2, we use Lemmas 3.1 and 3.2 to deduce a $L^{\infty}$ bound on certain moments of solutions. When there is fragmentation, our $L^{\infty}$ bounds are expressed in terms of suitable $L^{r}$-bounds. We first state our result in the discrete case.

Lemma 3.2 For every $r>1+\frac{d}{2}$ and $b \geq 0$, there exists a finite constant $C_{0}(r, T)$ such that for every $t \leq T$,

$$
\begin{aligned}
\operatorname{esssup}_{x}\left(\sum_{n=1}^{\infty} n d(n)^{d / 2} f_{n}(x, t)\right) \leq & d(1)^{d / 2} \operatorname{esssup}_{x}\left(\sum_{n=1}^{\infty} n f_{n}(x, 0)\right) \\
+ & C_{0}(r, T)\left(\sum_{n=1}^{\infty} n^{1-b} d(n)^{d-b_{0} d / 2}\right) \\
& \cdot\left(\int_{0}^{T} \int\left(\sum_{n} n^{b+a_{0}} f_{n}(x, s)\right)^{r} d x d s\right)^{1 / r}
\end{aligned}
$$

where $b_{0}=r /(r-1)$.

Proof. We note that the middle term on the right-hand side of (3.1) is non-positive because $\sum_{1}^{l} n Q_{n}^{C} \leq 0$. As for the fragmentation term, observe that $\int_{0}^{t} S_{t-s}^{D} h_{s} d s=p_{D} * h$ where the convolution is in both $x$ and $t$ variables and

$$
p_{D}(x, t)= \begin{cases}(4 \pi D t)^{-d / 2} \exp \left(\frac{-|x|^{2}}{4 D t}\right) & \text { if } t>0 \\ 0 & \text { if } t<0\end{cases}
$$

Note that

$$
\begin{aligned}
\int_{0}^{T} \int p_{D}(x, s)^{b_{0}} d x d s & =(4 \pi)^{\frac{d}{2}\left(1-b_{0}\right)} b_{0}^{-\frac{d}{2}} \int_{0}^{T}(D s)^{\frac{d}{2}\left(1-b_{0}\right)} d s \\
& =c_{1}\left(T, b_{0}\right) D^{\frac{d}{2}\left(1-b_{0}\right)}
\end{aligned}
$$

is finite if and only if $b_{0}<\frac{2}{d}+1$. Hence if $\frac{1}{b_{0}}+\frac{1}{r}=1$ with $b_{0}<\frac{2}{d}+1$ and $r>1+\frac{d}{2}$, then

$$
\left\|\int_{0}^{t} S_{t-s}^{d(n)} Q_{n}^{+, F}(s) d s\right\|_{L^{\infty}} \leq\left\|p_{d(n)}\right\|_{L^{b_{0}}}\left\|Q_{n}^{+, F}\right\|_{L^{r}}
$$




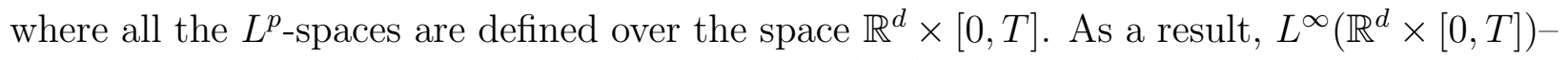
norm of the third term on the right-hand side of (3.1) is bounded above by

$$
\begin{aligned}
c_{1} \sum_{1}^{l} n d(n)^{d / 2} d(n)^{\frac{d}{2}\left(1-b_{0}\right)}\left\|Q_{n}^{+, F}\right\|_{L^{r}} & \leq c_{2} \sum_{1}^{l} n d(n)^{d-b_{0} d / 2}\left\|\sum_{m=1}^{l}(n+m)^{a_{0}} f_{n+m}\right\|_{L^{r}} \\
& \leq c_{2} \sum_{1}^{l} n^{1-b} d(n)^{d-b_{0} d / 2}\left\|\sum_{m=1}^{l}(n+m)^{a_{0}+b} f_{n+m}\right\|_{L^{r}} \\
& \leq c_{2}\left(\sum_{1}^{l} n^{1-b} d(n)^{d-b_{0} d / 2}\right)\left\|X_{a_{0}+b}\right\|_{L^{r}} .
\end{aligned}
$$

From this and (3.1) we deduce (3.6) because $\left\|S_{t}^{D} h\right\|_{L^{\infty}\left(\mathbb{R}^{d}\right)} \leq\|h\|_{L^{\infty}\left(\mathbb{R}^{d}\right)}$.

Remark 3.2. By a verbatim argument, we can show

$$
\begin{aligned}
\operatorname{essup}_{x}\left(\int_{0}^{\infty}(n+1) d(n)^{d / 2} f_{n}(x, t) d n\right) \leq & d(0)^{d / 2} \operatorname{esssup}_{x}\left(\int_{0}^{\infty}(n+1) f_{n}(x, 0) d n\right) \\
+ & C_{0}(r, T)\left(\int_{0}^{\infty}(n+1)^{1-b} d(n)^{d-b_{0} d / 2}\right) \\
& \cdot\left(\int_{0}^{T} \int\left(\int_{0}^{\infty}(n+1)^{b+a_{0}} f_{n}(x, s) d n\right)^{r} d x d s\right)^{1 / r},
\end{aligned}
$$

in the case of the continuous Smoluchowski equation.

Proof of Theorem 1.2. We first present the proof in the discrete case. To ease the notation, we simply write $L^{p}$ for $L^{p}\left(\mathbb{R}^{d} \times[0, T]\right)$. Set

$$
\hat{X}_{\theta}=\sum_{n} n^{\theta} d(n)^{d / 2} f_{n} .
$$

Given any real number $l>1$, we use Jensen's inequality to assert,

$$
\begin{aligned}
& \hat{X}_{\theta+1}=\sum_{n} n \frac{n d(n)^{d / 2} f_{n}}{\hat{X}_{1}} \hat{X}_{1}, \\
& \hat{X}_{\theta+1}^{l} \leq \hat{X}_{1}^{l-1} \sum_{n} n^{\theta l+1} d(n)^{d / 2} f_{n} .
\end{aligned}
$$

From this and Lemma 3.2 we deduce that for every $r>1+\frac{d}{2}$,

$$
\begin{aligned}
\left\|\hat{X}_{\theta+1}\right\|_{L^{l}} & \leq\left\|\hat{X}_{1}\right\|_{L^{\infty}}^{1-\frac{1}{l}}\left\|\hat{X}_{\theta l+1}\right\|_{L^{1}}^{\frac{1}{l}} \\
& \leq\left\{c_{1}+C_{0}(r, T)^{1-\frac{1}{l}}\left(\sum_{n} n^{1-b} d(n)^{d-b_{0} d / 2}\right)^{1-\frac{1}{l}}\left\|X_{b+a_{0}}\right\|_{L^{r}}^{1-\frac{1}{l}}\right\}\left\|\hat{X}_{\theta l+1}\right\|_{L^{1}}^{\frac{1}{l}},
\end{aligned}
$$


with $c_{1}$ a constant depending on the initial densities and $b_{0}=r /(r-1)$. Choose $r=l$ and choose $b$ so that

$$
\sum_{n} n^{1-b} d(n)^{d-b_{0} d / 2}=\sum_{n} n^{1-b} d(n)^{d(l-2) /(2 l-2)}<\infty .
$$

We are now assuming that $l>1+\frac{d}{2}$ and we choose any $\theta$ such that

$$
n^{\theta+1} d(n)^{d / 2} \geq n^{b+a_{0}}
$$

so that

$$
X_{b+a_{0}} \leq \hat{X}_{\theta+1}
$$

For such a pair of $\theta$ and $b$ we obtain

$$
\left\|\hat{X}_{\theta+1}\right\|_{L^{l}} \leq c_{2}+c_{2}\left\|\hat{X}_{\theta l+1}\right\|_{L^{1}}
$$

for a suitable constant $c_{2}$. In summary, for sufficiently large $\theta$, we always have (3.8) provided that $l>1+d / 2$. Hence $L^{l}$-norm of $\hat{X}_{\theta+1}$ is finite if $L^{1}$-norm of sufficiently large moment of $f$ is finite. Recall that we are assuming Hypothesis 1.2. To have (3.8), we may choose $\theta$ and $b$ any pair of numbers satisfying

$$
\theta \geq b+a_{0}+\frac{d}{2} b_{1}-1, \quad b>2-b_{2} d \frac{l-2}{2 l-2},
$$

provided that $l \geq 2$ and $l>1+d / 2$. Hence, given such a number $l$, we may assume that $\theta$ satisfies

$$
\theta>1+a_{0}+\frac{d}{2}\left(b_{1}-b_{2} \frac{l-2}{l-1}\right)
$$

in order to have (3.8). From this we deduce

$$
X_{a} \in L^{1} \Rightarrow X_{B} \in L^{\frac{2 a+b_{2} d-2}{2 B+b_{1} d-2}}
$$

provided that

$$
B>2-b_{2} d \frac{l-2}{2 l-2}+a_{0}=2+a_{0}-\frac{b_{2} d}{2}+\frac{b_{2} d}{2 l-2}, \quad l>\max \left\{1+\frac{d}{2}, 2\right\},
$$

with

$$
l=\frac{2 a+b_{2} d-2}{2 B+b_{1} d-2} .
$$

We note that the second condition implies the first one if we assume

$$
B \geq B_{0}:=2+a_{0}-b_{2}(1-d / 2)^{-} .
$$


Also note that by our Hypothesis 1.2 , we have that $b_{1} d-2>0$.

Let us write $X_{a}(N)=\sum_{n \geq N} n^{a} f_{n}$. We certaily have

$$
\begin{aligned}
Q_{n}^{C,+} & =\sum_{n_{1}+n_{2}=n} \alpha\left(n_{1}, n_{2}\right) f_{n_{1}} f_{n_{2}} \\
& \leq \sum_{n_{1}, n_{2}} \mathbb{1}\left(n_{1} \geq \frac{n}{2} \text { or } n_{2} \geq \frac{n}{2}\right) \alpha\left(n_{1}, n_{2}\right) f_{n_{1}} f_{n_{2}} \\
& \leq c_{1}\left[X_{1}(1) X_{0}\left(\frac{n}{2}\right)+X_{0}(1) X_{1}\left(\frac{n}{2}\right)\right]
\end{aligned}
$$

because $\alpha\left(n_{1}, n_{2}\right) \leq c_{0}\left(n_{1}+n_{2}\right)$ by assumption. Moreover

$$
\begin{aligned}
Q_{n}^{F,+} & =2 \sum_{m=1}^{\infty} \beta(n, m) f_{n+m} \\
& \leq c_{2} \sum_{m=1}^{\infty}(n+m)^{a_{0}} f_{n+m} \leq c_{2} X_{a_{0}}(n) .
\end{aligned}
$$

Hence, for any $K \geq 0$,

$$
\begin{aligned}
Q_{n}^{+} & \leq c_{3} n^{-K}\left[X_{1} X_{K}+X_{K+1} X_{0}+X_{a_{0}+K}\right] \\
\left\|Q_{n}^{+}\right\|_{L^{p}} & \leq c_{3} n^{-K}\left[\left\|X_{1}\right\|_{L^{l_{1}}}\left\|X_{K}\right\|_{L^{l_{2}}}+\left\|X_{K+1}\right\|_{L^{l_{3}}}\left\|X_{0}\right\|_{L^{l_{4}}}+\left\|X_{a_{0}+K}\right\|_{L^{p}}\right]
\end{aligned}
$$

provided that $\frac{1}{p}=\frac{1}{l_{1}}+\frac{1}{l_{2}}=\frac{1}{l_{3}}+\frac{1}{l_{4}}$. To bound the right-hand side, we would like to apply (3.9) with $B=0,1, K, K+1$ and $K+a_{0}$. Note that $B$ can not be smaller than $B_{0}$. So, we need to assume that $K \geq B_{0}^{+}$. However since in general we may not have $0 \geq B_{0}$, we need to choose $B=B_{0}^{+}$and $\left(B_{0}-1\right)^{+}+1$ in (3.9) in place of $B=0$ and $B=1$ respectively. Hence, if $X_{a} \in L^{1}$, then by (3.9),

$$
\begin{aligned}
& X_{0} \in L^{\frac{2 a+b_{2} d-2}{2 B_{0}^{+}+b_{1} d-2}}, \quad X_{1} \in L^{\frac{2 a+b_{2} d-2}{2\left(B_{0}-1\right)^{+}+b_{1} d}}, \quad X_{K} \in L^{\frac{2 a+b_{2} d-2}{2 K+b_{1} d-2}}, \\
& X_{K+1} \in L^{\frac{2 a+b_{2} d-2}{2 K+b_{1} d}}, \quad X_{a_{0}+K} \in L^{\frac{2 a+b_{2} d-2}{2 a_{0}+2 K+b_{1} d-2}},
\end{aligned}
$$

provided that $a$ and $K$ satisfy

$$
\frac{2 a+b_{2} d-2}{2 K+b_{1} d}, \frac{2 a+b_{2} d-2}{2 a_{0}+2 K+b_{1} d-2}>\max (2,1+d / 2), \quad K \geq B_{0}^{+} .
$$

From this we learn

$$
X_{a} \in L^{1} \Rightarrow\left\|Q_{n}^{+}\right\|_{L^{p}} \leq c n^{-K}
$$


provided that $a$ and $K$ satisfy (3.10) and $p$ is chosen to be

$$
p=\frac{2 a+b_{2} d-2}{2 K+b_{1} d-2+\max \left(2 B_{0}^{+}+b_{1} d, 2 a_{0}\right)} .
$$

We now follow [HR1]. We certainly have

$$
f_{n}(x, t)=\left(S_{t}^{d(n)} f_{n}^{0}\right)(x)+\left(p_{d(n)} * Q_{n}\right)(x, t) \leq\left(S_{t}^{d(n)} f_{n}^{0}\right)(x)+\left(p_{d(n)} * Q_{n}^{+}\right)(x, t) .
$$

So,

$$
\left\|f_{n}\right\|_{L^{\infty}} \leq\left\|\left(S_{t}^{d(n)} f_{n}^{0}\right)(x)\right\|_{L^{\infty}}+\left\|p_{d(n)}\right\|_{L^{r}}\left\|Q_{n}^{+}\right\|_{L^{p}}
$$

provided that $\frac{1}{r}+\frac{1}{p}=1$. Since by (3.7) we have $p_{D} \in L^{r}$ with $r<\frac{2}{d}+1$, it suffices to have

$$
\frac{1}{1+2 / d}+\frac{1}{p}<1
$$

or equivalently $p>1+d / 2$. With $p$ as in (3.12), it suffices to have

$$
\frac{2 a+b_{2} d-2}{2 K+b_{1} d-2+\max \left(2 B_{0}^{+}+b_{1} d, 2 a_{0}\right)}>\max (2,1+d / 2) .
$$

More precisely,

$$
K<K_{0}\left(a ; b_{1}, b_{2}, a_{0}\right):=\frac{a+b_{2} d / 2-1}{\max (2,1+d / 2)}-\frac{b_{1} d}{2}+1+\max \left(B_{0}^{+}+\frac{b_{1} d}{2}, a_{0}\right) .
$$

Note that this condition implies the first condition in (3.10). In summary, we need $K$ to satisfy (3.14) and $K \geq B_{0}^{+}$, and $r$ to satisfy $\frac{d}{2}(1-r)>-1$. (We remark that by our assumptions $a>1$ and $b_{1} d>2$, so (3.15) is compatible with the requirement $K \geq B_{0}^{+}$, because $K_{0}>B_{0}^{+}$.) Hence, if $X_{a} \in L^{1}, K \geq B_{0}^{+}$and $K$ satisfies (3.14), then

$$
\left\|f_{n}\right\|_{L^{\infty}} \leq A_{n}+c_{3} n^{-K} d(n)^{d(1-r) / 2} \leq A_{n}+c_{3} n^{-K} d(n)^{-1},
$$

where

$$
A_{n}=\left\|\left(S_{t}^{d(n)}\right) f_{n}^{0}(x)\right\|_{L^{\infty}} \leq\left\|f_{n}^{0}\right\|_{L^{\infty}\left(\mathbb{R}^{d}\right)} .
$$

As a result, we would have that $\sum_{n} n^{e}\left\|f_{n}\right\|_{L^{\infty}}<\infty$ if $X_{a} \in L^{1}$,

$$
\sum_{n} n^{e-K} d(n)^{-1}<\infty
$$

and,

$$
\sum_{n} n^{e}\left\|f_{n}^{0}\right\|_{L^{\infty}\left(\mathbb{R}^{d}\right)}<\infty
$$


For (3.15) it suffices to have

$$
e-K+b_{1}<-1 \text {. }
$$

Such a number $K$ can be found if we have

$$
e<\gamma\left(a ; b_{1}, b_{2}, a_{0}\right):=K_{0}\left(a ; b_{1}, b_{2}, a_{0}\right)-b_{1}+1
$$

In summary, if

$$
X_{a} \in L^{1}, \quad \sum_{n} n^{e}\left\|f_{n}^{0}\right\|_{L^{\infty}\left(\mathbb{R}^{d}\right)}<\infty, \quad e<\gamma\left(a ; b_{1}, b_{2}, a_{0}\right)
$$

then

$$
\sum_{n} n^{e}\left\|f_{n}\right\|_{L^{\infty}\left(\mathbb{R}^{d} \times[0, T]\right)}<\infty
$$

where

$$
\gamma\left(a ; b_{1}, b_{2}, a_{0}\right)=\frac{2 a+b_{2} d-2}{\max (4, d+2)}-b_{1}\left(1+\frac{d}{2}\right)+\max \left(B_{0}^{+}+\frac{b_{1} d}{2}, a_{0}\right) .
$$

We now turn to the proof in the the continuous case. The proof in this case is almost identical to the proof in the discrete case. The only step which requires an explanation is the fact that in the continuous case we can not assert that $X_{a} \leq X_{b}$ whenever $a \leq b$, because now all integrations are over $n \in(0, \infty)$. However, we can repeat all the steps if we replace $n$ with $n+1$. More precisely we switch from $X_{a}$ and $\hat{X}_{a}$ in the proof to $X_{a}^{\prime}=\int_{0}^{\infty}(n+1)^{a} f_{n} d n$, and $\hat{X}_{a}^{\prime}=\int_{0}^{\infty}(n+1)^{a} d(n)^{d / 2} f_{n} d n$. For this, we need to make sure that $X_{a}, X_{a_{0}} \in L^{1}$ imply that $X_{a}^{\prime} \in L^{1}$. This would follow if we can show

$$
\sup _{t} \int_{0}^{1} \int f_{n}(x, t) d x d n<\infty
$$

This can be readily establish because the expression

$$
\int_{0}^{1} f_{n}(x, 0) d n d x-\int_{0}^{1} f_{n}(x, t) d n d x
$$

equals

$$
\begin{aligned}
& \int_{0}^{t} \iiint[\mathbb{1}(n+m \leq 1)-\mathbb{1}(n \leq 1)-\mathbb{1}(m \leq 1)]\left(\alpha(m, n) f_{n} f_{m}-\beta(m, n) f_{n+m}\right) d m d n d x d s \\
& \leq 2 \int_{0}^{t} \iiint \mathbb{1}(n \text { or } m \leq 1) \beta(m, n) f_{n+m} d m d n d x d s \leq 4 c_{0} X_{a_{0}},
\end{aligned}
$$

where we have used Hypothesis 1.3 for the second inequality. This completes the proof. 


\section{References}

[A] H. Amann, Coagulation-fragmentation processes, Arch. Rational Mech. Anal. 339-366, $151(2000)$.

[AW] H. Amann and Ch. Walker, Local and global strong solutions to continuous coagulation-fragmentation equations with diffusion, J. Differential Equations, 159-186, 218 (2005) .

[BC] J. M. Ball and J. Carr, The discrete coagulation-fragmentation equations: existence, uniqueness, and density conservation, J. Statist. Phys., 203-234, 61, (1990).

[HR1] A. M. Hammond and F. Rezakhanlou, Moment bounds for Smoluchowski Equation and their consequences, Commun. Math. Phys., 645-670 276 (2008).

[HR2] A. M. Hammond and F. Rezakhanlou, The kinetic limit of a system of coagulating Brownian particles, Arch. Rational Mech. Anal., 1-67, (2007).

[HR3] A. M. Hammond and F. Rezakhanlou, Kinetic limit for a system of coagulating planar Brownian particles, J. Stat. Phys., 997-1040, 124, (2006).

[LM1] Ph. Laurençot and S. Mischler, Global existence for the discrete diffusive coagulationfragmentation equations in $L^{1}$, Rev. Mat. Iberoamericana, 731-745, 18 (2002).

[LM2] Ph. Laurençot and S. Mischler, The continuous coagulation-fragmentation equations with diffusion, Arch. Ration. Mech. Anal. 45-99, 162 (2002).

[MR] S. Mischler and M. Rodriguez Ricard, Existence globale pour l'equation de Smoluchowski continue non homogene et comportement asymptotique des solutions, C. R. Acad. Sci. Paris, Ser. I Math. 407-412, 336 (2003) .

[N] J.R. Norris, Brownian coagulation, Comm. Math. Sci. 93-101, 1 (2004) .

[W1] D. Wrzosek, Weak solutions to the Cauchy problem for the diffusive discrete coagulation-fragmentation system, J. Math. Anal. Appl.,405-418,289(2004).

[W2] D. Wrzosek, Mass-conserving solutions to the discrete coagulation-fragmentation model with diffusion, Nonlinear Anal. 297-314,49 (2002).

[W3] D. Wrzosek, Existence of solutions for the discrete coagulation-fragmentation model with diffusion, Topol. Methods Nonlinear Anal. 279-296, 9 (1997) .

[YRH] M. R. Yaghouti, F. Rezakhanlou and A. M. Hammond, Coagulation, diffusion and continuous Smoluchowski equation, preprint. 